\title{
Static characteristics of the double rotor switched reluctance motor
}

\begin{abstract}
Static measurement characteristics of the double rotor switched reluctance motor developed on the basis of double salient dual air-gap structure is put forward in this paper. The reduction of the air - gap length of the conventional machine is constrained by the mechanical intolerances that inherit the torque generating capability. Therefore there is always a limitation on the air-gap length. However, with reduced air-gap length the magnetic flux control is improved with the aid of dual air-gap structure inside the machine. Analytical and finite element analysis is carried out to investigate the magnetic field distribution at different rotor positions and with different currents. The static torque characteristics derived from the fabricated model is compared with that of the finite element methods and analytical methods. The measurement results are in closer agreement with the analytical and simulation. Motor constant square density is used for the performance evaluation of the proposed machine and the characteristics are evaluated and compared by all three methods presented.
\end{abstract}

Keyword: Double rotor; Reluctance; FEM; Motor constant square density 